\title{
The Effect of Molecular Weight of Polycaprolactone on the Ester Interchange Reactions during Melt Blending with Poly(ethylene terephthalate)
}

\author{
Kyung Yul LIM, ${ }^{\dagger}$ Byoung Chul KIM, and Kee Jong YooN* \\ Polymer Structuring Laboratory, Department of Fiber and Polymer Engineering, Division of Chemical Engineering, \\ Hanyang University, 17 Haengdang, Seongdong, Seoul 133-791, Korea \\ ${ }^{*}$ Department of Textile Engineering, College of Engineering, Dankook University, \\ San 8 Hannam, Yong-san, Seoul 140-714, Korea
}

(Received July 27, 2001; Accepted March 2, 2002)

\begin{abstract}
The molecular weight (MW) of polycaprolactone (PCL) component had a profound effect on the ester interchange reactions during melt blending with poly(ethylene terephthalate) (PET). Utilization of lower MW of PCL component gave rise to higher degree of ester interchange reactions, and it could reduce mixing time required for generating a copolyester. The thermal properties and crystalline structure of resultant copolyesters were depended on the degree of ester interchange reactions by varying MW of PCL. It converted the morphology of copolyesters to a continuous single phase, suggesting formation of more random copolyester. Further, the substitution of PCL with low MW $\left(M_{\mathrm{w}}: 1250\right)$ as third component in melt blends of PET and PCL with high MW( $\left.M_{\mathrm{w}}: 80000\right)$ led to higher degree of ester interchange reactions as well.

KEY WORDS Poly(ethylene terephthalate)/ Polycaprolactone / Melt Blending / Ester Interchange Reactions / Molecular Weight /
\end{abstract}

The ester interchange reactions commonly occur in melt blending of different kinds of polyesters, which produces first the block copolyesters from immiscible blends and finally microstructure becomes randomized. ${ }^{1-4}$ The physicochemical properties of produced copolyesters are closely related to their structural characteristics such as sequence length and randomness, which influenced by the degree of ester interchange reactions. Therefore, the control of ester interchange reactions in polyester blends may be technical key for their industrial applications.

On the other hand, the copolyesters consisted of amorphous aliphatic ester unit and high melting crystalline ester unit are expected to find their applications for functional materials. Among them, poly(ethylene terephthalate/caprolactone) $[\mathrm{P}(\mathrm{ET} / \mathrm{CL})]$ copolyester has been early focused on the biodegradable polymeric materials required for desirable mechanical and thermal properties. ${ }^{5-10}$ It has recently attracted much interest for elastomeric materials. ${ }^{11,12}$ Thus, the elastic fiber spun from $\mathrm{P}(\mathrm{ET} / \mathrm{CL})$ copolyesters is used as plastic and mechanical energy-absorbing device in seat belt assembly.

However, it is difficult to obtain the copolyester by melt blending of poly(ethylene terephthalate) (PET) and polycaprolactone (PCL) because of the poor thermal stability of PCL. In order to prevent the thermal degradation of PCL component during melt blending, the mixing procedure must be accomplished within a short time. However, this frequently raises the problem of immiscibility between PET and PCL because of the lack of ester interchange reactions.

As well known, ester interchange reactions during melt blending includes alcoholysis, acidolysis, and direct transesterification. ${ }^{4,13-15}$ The degree of ester interchange reactions is more seriously dependent on alcoholysis than acidolysis and direct transesterification because the former reaction is kinetically faster than the latter reactions. Recently, it is reconfirmed the fact that the importance of the role of alcoholysis by hydroxyl end group via the control of molecular weight (MW) of PET in the kinetics of the ester interchange reactions between PET and poly(ethylene naphthalene-2,6dicarboxylate) (PEN). ${ }^{16-18}$

Taking into account the aforementioned reports, one of the possible solutions for the problems in melt blending of PET and PCL may be the control of the MW of PCL component. Thus, lower MW of PCL component can lead to higher degree of ester interchange reactions in short blending time because it increases the concentration of hydroxyl end group as presented in Figure 1. Consequently, this can minimize the thermal degradation of PCL in the melt blending procedure.

The principal objective of this study is to examine the effect of MW of PCL on reactivity between PET and PCL during melt blending. We attempt to increase

${ }^{\dagger}$ To whom correspondence should be addressed (Tel : +82-2-2290-0494, Fax : +82-2-2297-4941, E-mail: legal@unitel.co.kr). 


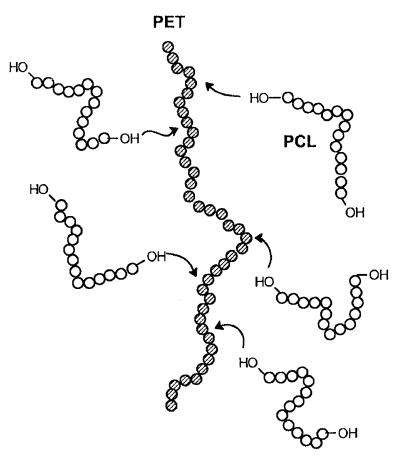

(a)

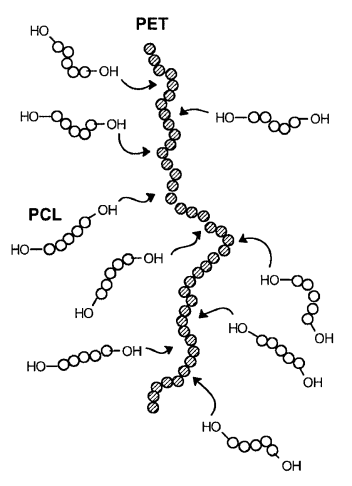

(b)
Figure 1. The effect of molecular weight of PCL on the ester interchange reactions of PET/PCL blend; (a) high molecular weight, (b) low molecular weight.

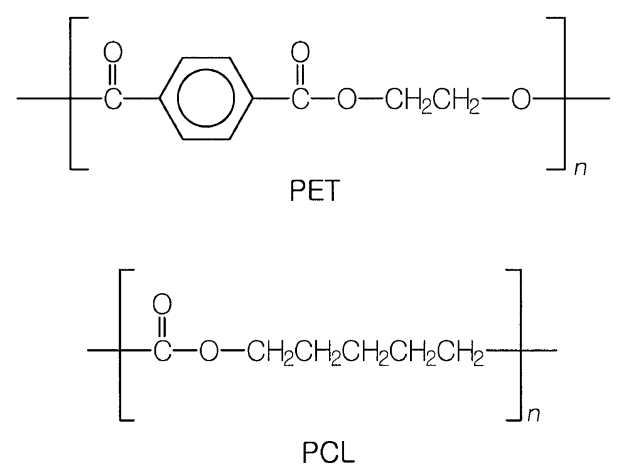

Figure 2. The chemical structures of PET and PCL.

the reactivity between PET and PCL for short blending time through two preparative methods as follows; (1) the variation of MW of PCL component in melt blending of PET and PCL, (2) the substitution of PCL with low MW as third component in melt blending of PET and PCL with high MW. The ester interchange reactions between PET and PCL are characterized, and thermal properties, morphology and crystalline structure of resultant copolyesters are discussed as well.

\section{EXPERIMENTAL}

\section{Materials}

Commercial grade PET $\left(M_{\mathrm{w}}: 18000\right)$ and PCL $\left(M_{\mathrm{w}}\right.$ : $80000,40000,10000,1250$, and 530) were the base materials whose chemical structure shown in Figure 2. PCL were supplied by Aldrich Chemical Co., Polysciences Inc., and Union Carbide Co. Reagent grade tetrahydrofuran, $d$-trifluoroacetic acid and $\mathrm{CDCl}_{3}$ were used as received.

\section{Blends Preparation}

All samples were prepared by melt blending in a Haake mixer at $285^{\circ} \mathrm{C}$ for $10 \mathrm{~min}$. To examine the effect of MW of PCL, 65/35 (by wt) PET/PCL blends was prepared using PCLs with $M_{\mathrm{w}}: 80000,40000,10000$, 1250 , and 530, respectively. To examine the effect of the substitution of PCL with low MW, 50/50 (by wt) PET/PCL blends were prepared first using PCL with $M_{\mathrm{w}}: 80000$, and then PCL with $M_{\mathrm{w}}: 1250$ was added into $1,2,3,4,6,8$, and $10 \%$ (by wt) in place of PCL with $M_{\mathrm{w}}: 80000$, respectively.

\section{Measurements}

${ }^{1} \mathrm{H}$ NMR spectra were obtained by Varian Gemini 200 spectrometer $(200 \mathrm{MHz}$, U. S. A.) using $1 / 1(\mathrm{v} / \mathrm{v})$ $d$-trifluoroacetic acid/ $\mathrm{CDCl}_{3}$ mixture as the solvent. DSC curves were obtained by TA Instruments DSC 2100 (U. S. A) at the heating rate of $10^{\circ} \mathrm{C} \mathrm{min}^{-1}$ under nitrogen purging. The morphology of blends was observed by scanning electron microscopy (SEM ABT SX-40A, Japan). SEM samples were prepared by cryofracture with liquid nitrogen followed by etching the fractured surface in THF for $1 \mathrm{~min}$ to eliminate the PCL component. X-Ray diffractograms were obtained by wide angle X-Ray diffractometry (MacScience M18 , Japan) equipped with $\mathrm{Ni}$-filtered $\mathrm{Cu}-K_{\alpha}$ radiation at $45 \mathrm{kV}$ at $200 \mathrm{~m} \AA$. The weight of average molecular weight of the blends were measured by gel permeation chromatograpy (GPC, Waters Co.) using hexafluoroisopropanol $(0.2 \% \mathrm{w} / \mathrm{v})$ as the solvent at $23^{\circ} \mathrm{C}$, whose calibration curve was obtained by using monodisperse polystyrene.

\section{RESULTS AND DISCUSSION}

Figure 3 shows the ${ }^{1} \mathrm{H}$ NMR spectra of $65 / 35$ (by wt) PET/PCL blends prepared by using various PCLs with different MWs. Peaks (1) and (2) at chemical shifts 4.9 and $4.3 \mathrm{ppm}$ are assigned to the protons of ethylene glycol (EG) unit of PET and $-\mathrm{CO}-\mathrm{O}-\mathrm{CH}_{2}-$ of PCL, respectively. The peak (3) at chemical shift $4.1 \mathrm{ppm}$ may be assigned to the hydroxyl end groups of PCL. These new peaks around 4.9-4.4 ppm are assigned to the methylene hydrogen adjacent to the ester group whose chemical environments are different from those in PET and PCL. The appearance of new peaks on the ${ }^{1} \mathrm{H}$ NMR spectra suggests that $\mathrm{P}(\mathrm{ET} / \mathrm{CL})$ copolyesters are indeed formed through ester interchange reactions during melt blending.

These new peaks are not noticeable in blends using PCL with $M_{\mathrm{w}}$ higher than 10000 but obviously noticed in blends using PCL with $M_{\mathrm{w}}: 1250$ and 530, suggesting that PCL with lower MW gives rise to higher degree of ester interchange reactions. This fact may be explained by the increase of the concentration of hydroxyl end groups and the chain mobility of PCL at the molten state. In other words, a low MW of PCL $\left(M_{\mathrm{w}}: 1250\right.$ 


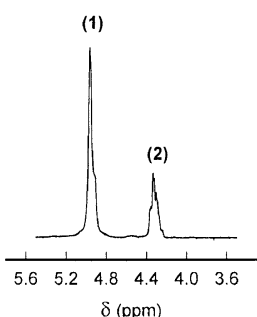

(a)

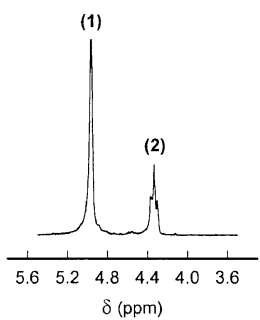

(b)

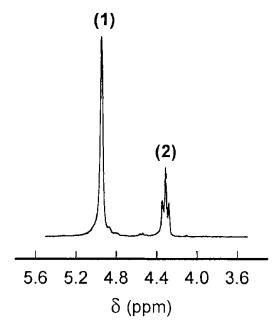

(c)

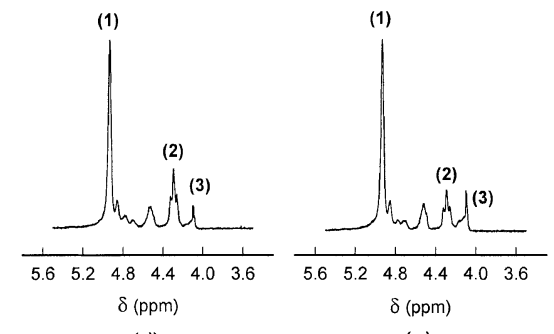

(d)

(e)
Figure 3. $\quad{ }^{1} \mathrm{H}$ NMR spectra of PET/PCL (65/35 by wt) blends prepared by using various PCLs with different MWs; (a) $M_{\mathrm{w}}$ : 80000 , (a) $M_{\mathrm{w}}: 40000$, (a) $M_{\mathrm{w}}: 10000$, (a) $M_{\mathrm{w}}: 5000$, (a) $M_{\mathrm{w}}$ : 1250 .

and 530) would attack the ester groups of PET more readily than a high MW of PCL (over $M_{\mathrm{w}}: 10000$ ) because they have higher chain mobility and greater population of hydroxyl end groups. Thus, it is possible to control the reactivity of ester interchange between PET and PCL by varying MW of PCL. The ester interchange reactions between PET and PCL gave a result similar to PET and PEN blend systems. ${ }^{16-18}$

On the other hand, if ester interchange in the PET/PCL blend is only done by direct transesterification, the degree of ester interchange reactions should be identical because the population of ester groups in the blends is the same regardless of the MW of PCL at given composition. In fact, however, the degree of ester interchange reactions in PET/PCL blends is influenced by the MW of PCL. This means that the ester interchange reactions during melt blending are more seriously dependent on alcoholysis than direct transesterification.

Figures $4 \mathrm{a}$ and $\mathrm{b}$ show melting and crystallization behavior of $65 / 35$ (by wt $\%$ ) PET/PCL blends prepared by using various PCLs with different MW. The blends using PCLs with $M_{\mathrm{w}}$ higher than 10000 exhibit melting and crystallization peaks of ET and CL components, respectively. The melting and crystallization temperatures of ET and CL components remain unchanged but the heats of fusion and crystallization decrease with decreasing MW of PCL. The blends using PCLs with $M_{\mathrm{w}}$ : 1250 and 530 exhibit almost single peaks of ET block and melting and crystallization temperatures decrease with decreasing MW of PCL. This indicates that the
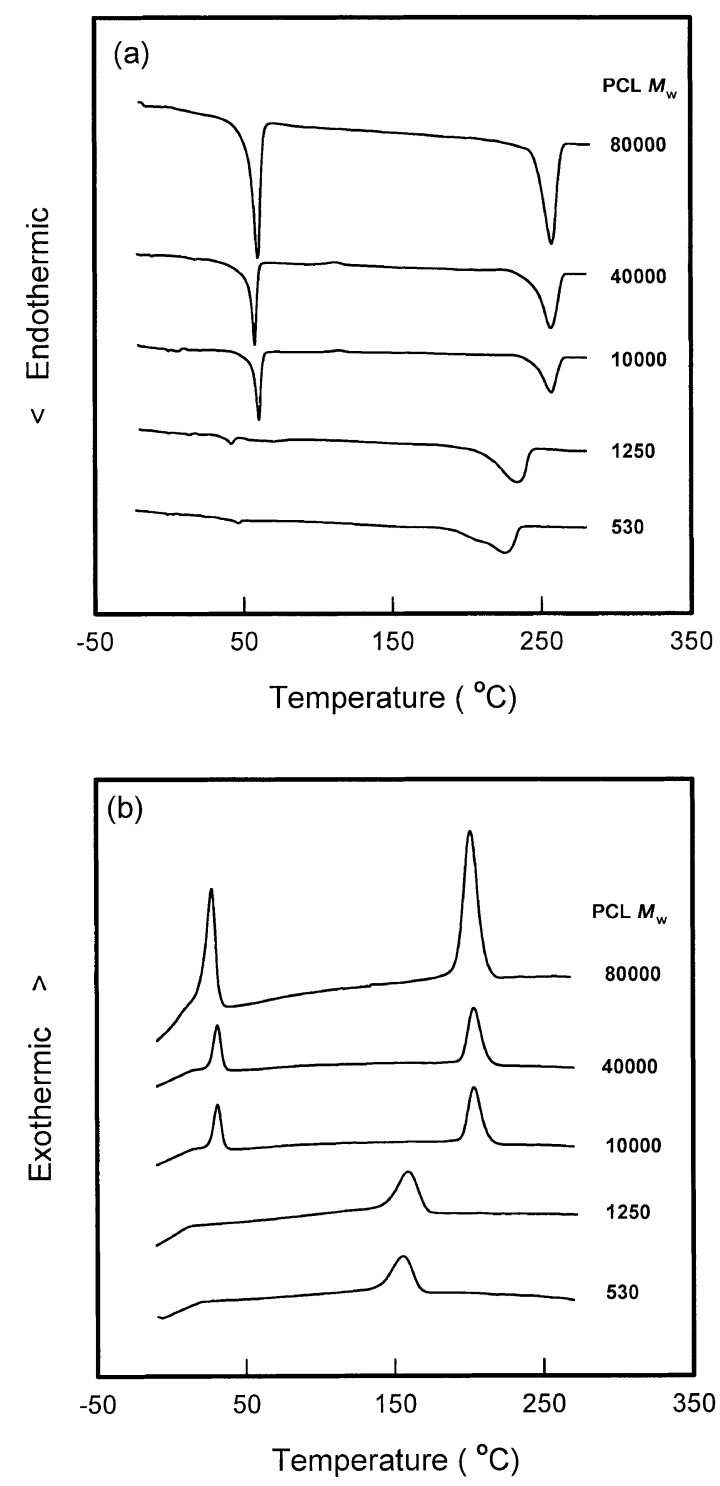

Figure 4. Melting and Crystallization behaviors of PET/PCL (65/35 by wt) blends prepared by using various PCLs with different MWs.

PET/PCL blends using low MW PCL produce random copolyesters whose CL block is not sufficiently long enough to crystallize.

As already mentioned in Figure 3, the PET/PCL blends containing PCL with $M_{\mathrm{w}}$ higher than 10000 make little difference in the degree of ester interchange reactions between PET and PCL for short blending time (within $10 \mathrm{~min}$ ). In order to examine the effect of $\mathrm{MW}$ of PCL on these PET/PCL blends using PCLs with $M_{\mathrm{w}}$ higher than 10000 , these blends were heat-treated in the DSC pan at $285^{\circ} \mathrm{C}$ for 10,20 , and $30 \mathrm{~min}$, respectively. As heat-treatment time is increased, the melting behavior of CL block gradually disappears and the melting temperature of ET block decreases as shown in Figure 5. Variation of melt temperature of ET block in PET/PCL blends with heat-treatment time is shown in Figure 6. In the PET/PCL blend containing PCL with 


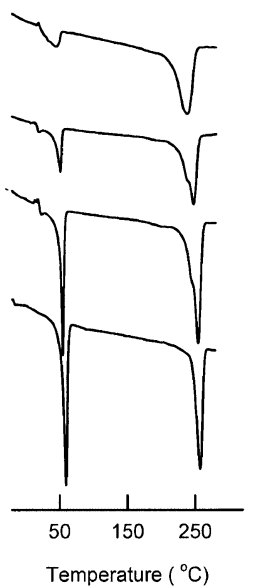

(a)

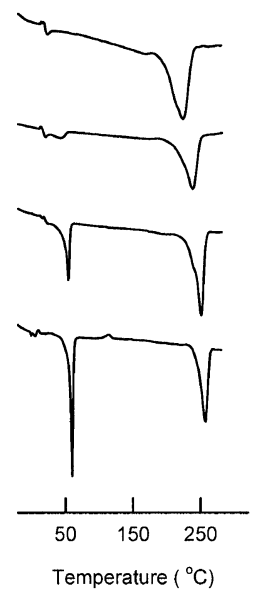

(b)

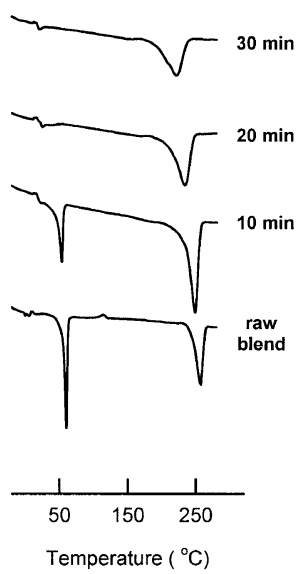

(c)
Figure 5. Melting behavior of PET/PCL (65/35 by wt) blends as a function of heat treatment time; (a) $M_{\mathrm{w}}: 80000$, (b) $M_{\mathrm{w}}: 40000$, (c) $M_{\mathrm{w}}: 10000$.

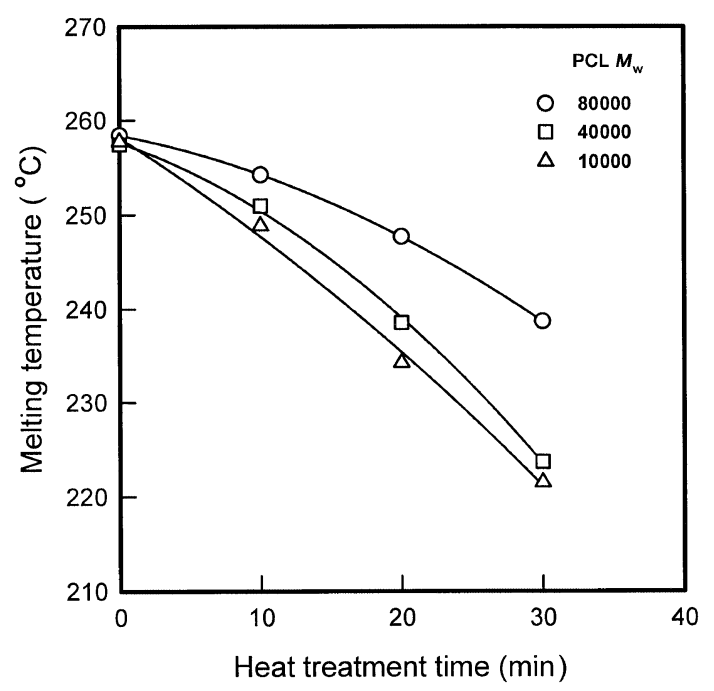

Figure 6. Variation of melting temperature of ET component as a function of heat treatment time in PET/PCL (65/35 by wt) blends prepared by using PCLs with $M_{\mathrm{w}}: 80000,40000$, and 10000 of PCL.

$M_{\mathrm{w}} 10000$, the melting temperature of ET blocks decreases more steeply than the blends using PCLs with $M_{\mathrm{w}}: 80000$ and 40000 , suggesting that the degree of ester interchange reactions in PET/PCL blend using PCLs with $M_{\mathrm{w}}$ higher than 10000 may be also dependent on MW of PCL.

Figure 7 shows the SEM micrographs of the THFetched surfaces of 65/35 (by wt) PET/PCL blends prepared by using various PCLs of different MWs. The soluble component etched from the blends is supposed as follows; (1) pure PCL unreacted with PET, (2) the copolyesters including the long CL block, (3) the random copolyesters composed of short ET and CL blocks. In the case of PET/PCL blends using PCLs with $M_{\mathrm{w}}$ higher than 10000 , the etched-out component produces craters. As MW of PCL is decreased, the

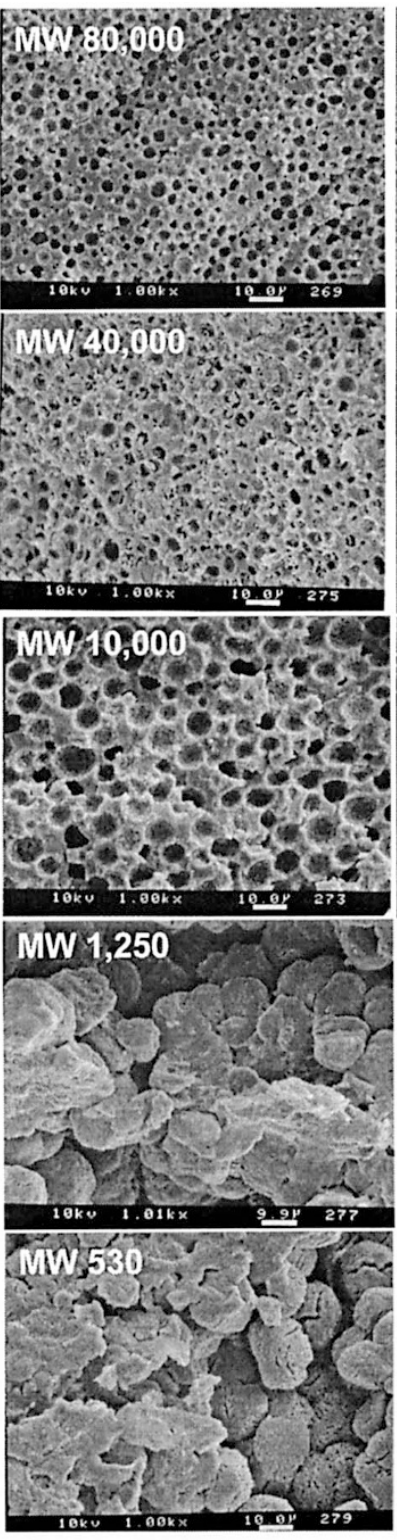

(a)

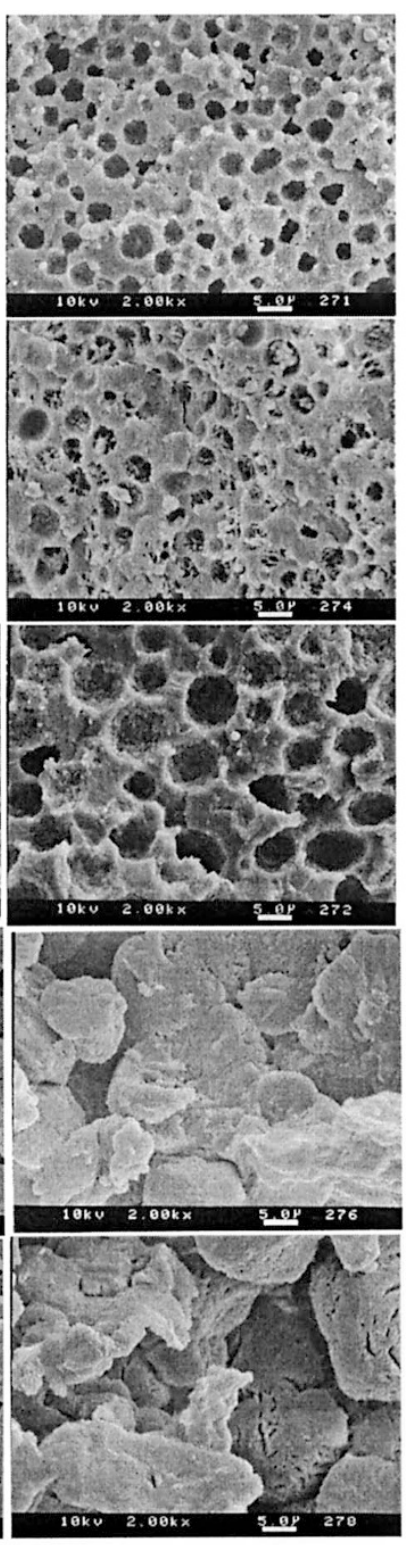

(b)
Figure 7. SEM micrographs of THF etched fracture surface of PET/PCL (65/35 by wt) blends prepared by using various PCLs with different MWs; (a) X:1000, (b) X:2000.

craters gradually disappear and finally morphology of the copolyesters changes to a continuous single phase. This also indicates that the $\mathrm{P}(\mathrm{ET} / \mathrm{CL})$ copolyester converts from heterogeneous block copolymer to homogeneous random copolymer with increasing the degree of ester interchange reactions by decreasing MW of PCL. As shown in Figure 8, the PET/PCL blends using PCLs with $M_{\mathrm{w}}$ higher than 10000 produce larger craters and coarser crater surface when MW of PCL is lower. This suggests that interfacial adhesion is enhanced with increasing the degree of ester interchange reactions. This result agrees well with the result of heat-treatment described in Figure 6.

Figure 9 shows the X-Ray diffraction patterns of the films of PET/PCL blends prepared by using various 


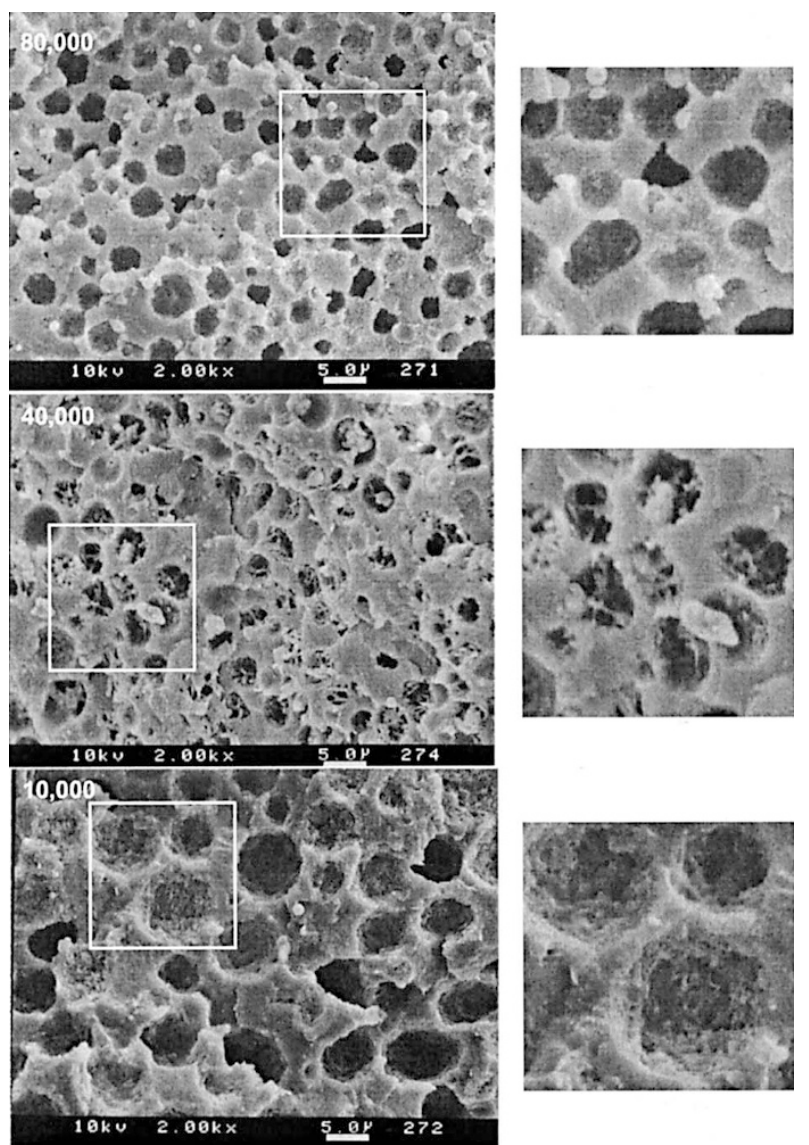

Figure 8. SEM micrographs of THF etched fracture surface of PET/PCL (65/35 by wt) blends prepared by using PCL with $M_{\mathrm{w}}$ : 80000,40000 , and 10000 .

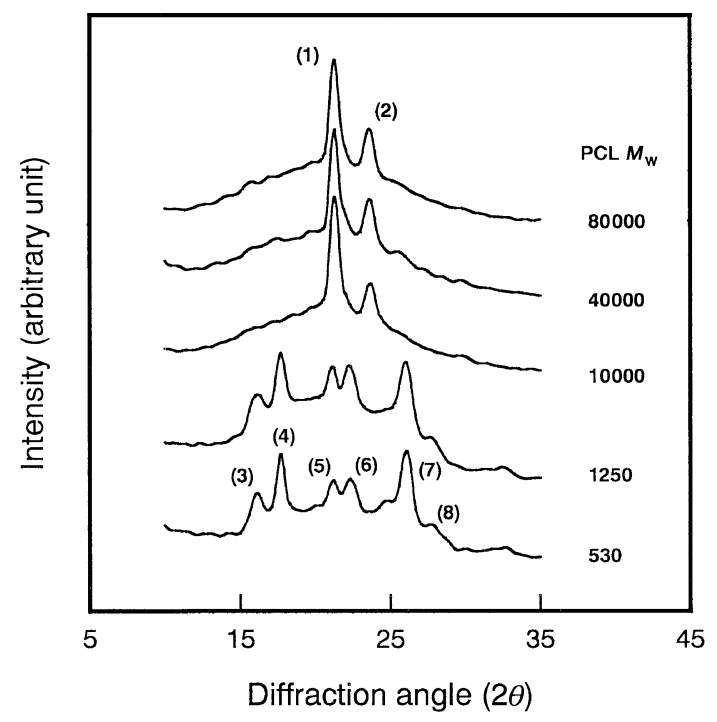

Figure 9. X-Ray diffraction patterns of PET/PCL (65/35 by wt) blends prepared by using various PCLs with different MW.

PCLs with different MW. The film samples were fabricated by melt casting in nitrogen purging. The peaks (1) and (2) are assigned to (110) and (200) planes of PCL, respectively. The peaks (3), (4), (5), (6), (7), and (8) are assigned to $(0-11),(010),(-111),(-110),(011)$, $(100)$, and $(1-11)$ planes of PET, respectively. Refer-

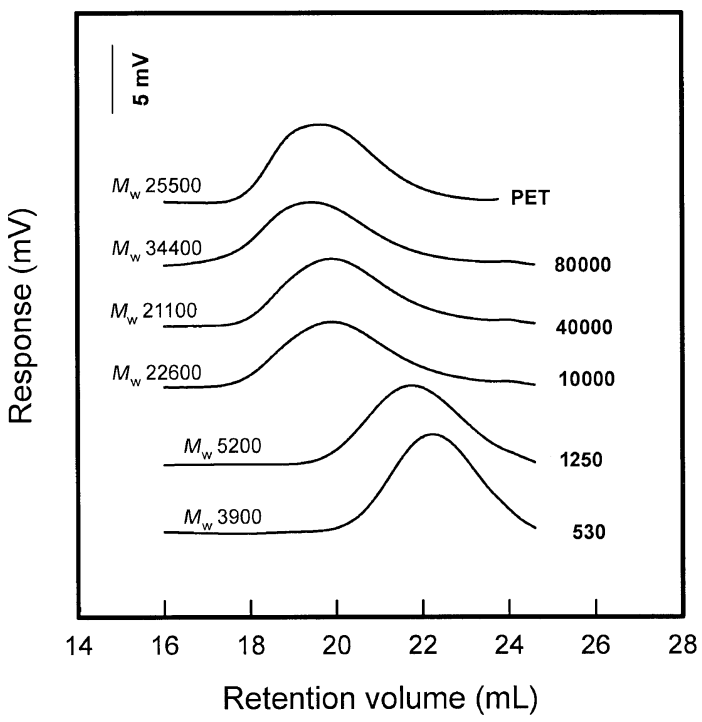

Figure 10. GPC curves of PET and PET/PCL (65/35 by wt) blends prepared by using various PCLs with different MW.

ring to DSC curves in Figure 4, the PET/PCL blends using PCLs with $M_{\mathrm{w}}$ higher than 10000 must exhibit the diffraction patterns of both ET and PL components. However, they exhibit only the diffraction pattern of CL component. It is recognized that the crystal of ET may be small and imperfect because ET component crystallizes much more slowly than $\mathrm{CL}$ component. In the case of PET/PCL blends using PCLs with $M_{\mathrm{w}}: 1250$ and 530, the X-Ray diffraction patterns exhibit only the diffraction pattern of ET component although both ET and CL components are included in the copolyesters. This suggests that CL component cannot crystallize because block length is decreased by the higher degree of ester interchange reactions.

In spite of high reactivity to ester interchange reactions, the PET/PCL blends using PCLs with low MWs exhibit poor mechanical properties. It can be explained by the reduction of $M_{\mathrm{w}}$. Figure 10 shows the GPC curves of PET and PET/PCL blends prepared by using various PCLs with different MWs, in which the $M_{\mathrm{w}}$ of samples determined by GPC is denoted. The $M_{\mathrm{w}}$ of PET/PCL blends using PCLs with $M_{\mathrm{w}}$ higher than 10000 is similar to that of PET homopolymer. However, The $M_{\mathrm{w}}$ of blends using PCLs with $M_{\mathrm{w}}: 1250$ and 530 decreases more seriously. Decreasing $M_{\mathrm{w}}$ deteriorates the mechanical properties of the resultants. From this result, we recommends that the PET/PCL blends is accomplished by using PCLs whose $M_{\mathrm{w}}$ is higher than 10000 for industrial applications.

In this case, however, the resultants exhibit immiscibility due to the lack of ester interchange reactions. In order to obtain the PET/PCL blends having a good combination of miscibility and mechanical properties, low MW of PCL (PCLLMW, $M_{\mathrm{w}}: 1250$ ) as third compo- 


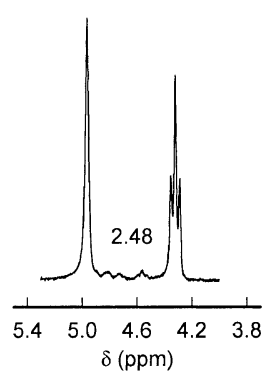

no addition

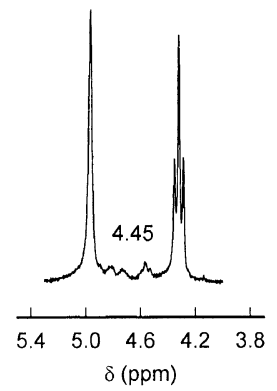

$4 \%$

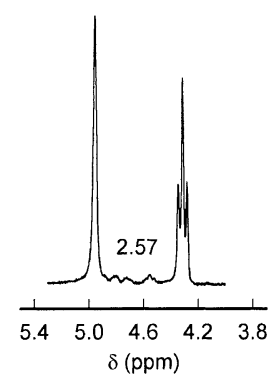

$1 \%$

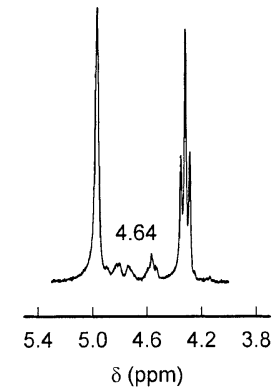

$6 \%$

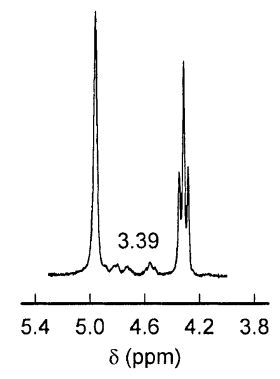

$2 \%$

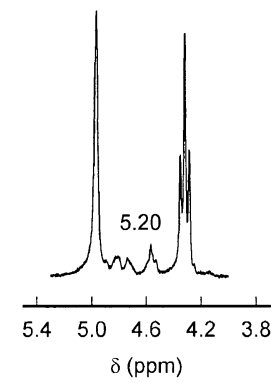

$8 \%$

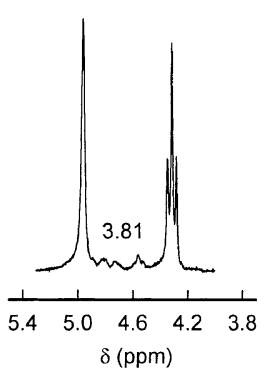

$3 \%$

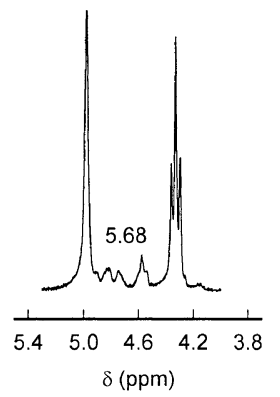

$10 \%$

Figure 11. ${ }^{1} \mathrm{H}$ NMR spectra of PET/PCL blends containing different content of $\mathrm{PCL}_{\mathrm{LMW}}$.
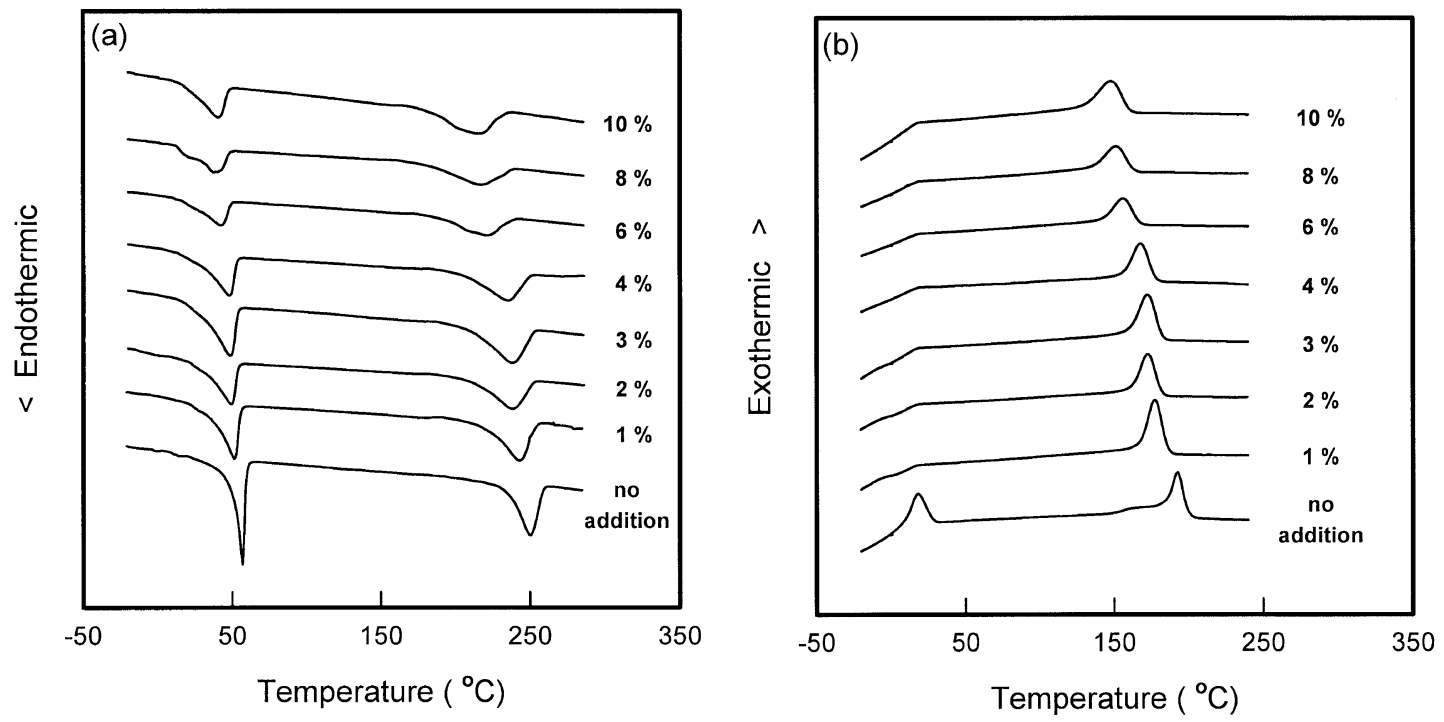

Figure 12. Melting and crystallization behaviors of PET/PCL blends containing different content of $P C L_{L M W}$.

nent is added to the PET/PCL blends using high MW of PCL (PCL $\left.\mathrm{HMW}_{\mathrm{W}}, M_{\mathrm{w}}: 80000\right)$. If only a small amount of copolyester is formed by ester interchange reactions between PCL $\mathrm{LMW}_{\mathrm{LM}}$ and PET, it can play a role of compatibilizer in the interface of unreacted PET and PCL $\mathrm{PMW}_{\mathrm{HM}}$.

Figure 11 shows the ${ }^{1} \mathrm{H}$ NMR spectra of

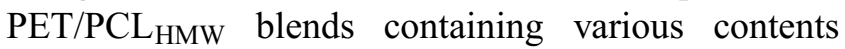
of $P C L_{L M W}$ and the number in the figure denotes the percent ratio of the area of new peaks based on the total area of all peaks. The new peaks around 4.9-4.4 ppm are a direct evidence of formation of copolyester by ester interchange reactions.

At higher content of $\mathrm{PCL}_{\mathrm{LMW}}$, the area of new peaks tends to be larger, indicating that reactivity to ester in- terchange reactions in the PET/PCL blends is increased by the active hydroxyl end groups of low MW PCL. As already mentioned in Figure 3, the peak at chemical shift $4.1 \mathrm{ppm}$ assigned to the hydroxyl end groups of PCL is not observed. This indicates that most of $\mathrm{PCL}_{\mathrm{LMW}}$ may participate in ester interchange reactions.

Figures 12 (a) and (b) show the melting and crystallization behaviors of the PET/PCL blends containing various contents of $P C L_{L M W}$. The melt and crystallization of temperatures and heats of ET component are decreased with increasing PCL $\mathrm{LMW}_{\mathrm{LM}}$ content as shown in Figure 13. This suggests that the sequence lengths of ET and CL blocks are decreased by higher reactivity to ester interchange reactions. Thus, the degree of ester 


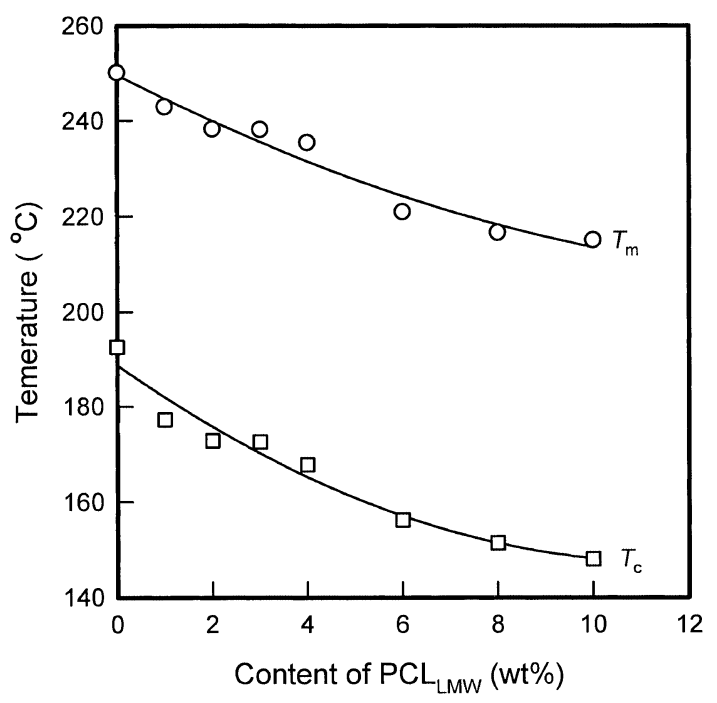

Figure 13. Variation of melting and crystallization temperatures of ET component in PET/PCL blends containing different content of $\mathrm{PCL}_{\mathrm{LMW}}$.

interchange reactions on PET/PCL melt blending can be controlled by adding low MW of PCL.

\section{CONCLUSION}

This study investigated the effect of the molecular weight of PCL component on ester interchange reactions between PET and PCL during melt blending. Utilization of PCL component of lower molecular weight gave rise to higher degree of ester interchange reactions. The substitution of PCL with low molecular weight $\left(M_{\mathrm{w}}: 1250\right)$ as third component in melt blends of PET and PCL with high molecular weight $\left(M_{\mathrm{w}}\right.$ : 80000 ) also increased the reactivity of the reactions. Since the degree of the ester interchange reactions during melt blending determines the structural characteristics of $\mathrm{P}(\mathrm{ET} / \mathrm{CL})$ copolyesters, their physicochemical properties could be regulated by utilizing PCL of properly selected molecular weight. That is, generating $\mathrm{P}(\mathrm{ET} / \mathrm{CL})$ copolyesters to improve the miscibility may be controlled by proper choice of molecular weight of PCL. This would be helpful in preparing PET/PCL blends with improved mechanical properties because of the properly controlled blending time, which can minimize the thermal decomposition of PCL during melt blending. These preparative methods may be extended to other melt blending systems of aliphatic polyesters and high melting polyesters as well.

\section{REFERENCES}

1. S. C. Lee, K. H. Yoon, I. H. Kim, H. C. Kim, and T. W. Son, Polymer, 38, 4831 (1997).

2. K. G. Gravalos, J. K. Kallitsis, and N. K. Kalfoglou, Polymer, 36, 1393 (1995).

3. A. Legros, P. J. Carreau, B. D. Favis, and A. Michel, Polymer, 35, 758 (1994).

4. R. S. Porter and L. H. Wang, Polymer, 33, 2019 (1992).

5. Y. Tokiwa and T. Suzuki, J. Appl. Polym. Sci., 26, 441 (1981).

6. Y. Tokiwa, T. Ando, T. Suzuki, and T. Takeda, Polym. Mater. Sci. Eng., 62, 988 (1990).

7. A. Iwamoto and Y. Tokiwa, Polym. Degrad. Stab., 45, 205 (1994).

8. H. S. Jun, B. O. Kim, Y. C. Kim, H. N. Chang, and S. I. Woo, J. Environ. Polym. Degrad., 2, 9 (1994).

9. H. S. Jun, B. O. Kim, H. N. Chang, and S. I. Woo, "Biodegradable Plastics and Polymers", H. Feng, Y. Han, and L. Huang, Ed., Elsevier Science Publishers B.V., Amsterdam, 1994, pp 498-504.

10. E. Chiellini, A. Corti, A. Giovannini, P. Narducci, M. Paparella, and R. Solaro, J. Environ. Polym. Degrad., 4, 37 (1996).

11. W. Tang, N. S. Murthy, F. Mares, M. E. McDonnel, and S. A. Curran, J. Appl. Polym. Sci., 74, 1858 (1999).

12. W. Tang, F. Mares, and R. C. Morgan, U. S. Patent 5830811 (Nov. 3, 1998).

13. A. M. Kotliar, J. Polym. Sci., Macromol. Rev., 16, 367 (1981).

14. F. Pilati, E. Marianucci, and C. Berti, J. Appl. Polym. Sci., 30, 1267 (1985).

15. D. Kint and S. M. Guerra, Polym. Int., 48, 346 (1999).

16. S. Collins, A. M. Kenwright, C. Pawson, S. K. Peace, and R. W. Richards, Macromolecules, 33, 2974 (2000).

17. A. M. Kenwright, S. K. Peace, R. W. Richards, A. Bunn, and W. A. Macdonald, Polymer, 40, 2035 (1999).

18. A. M. Kenwright, S. K. Peace, R. W. Richards, A. Bunn, and W. A. Macdonald, Polymer, 40, 5851 (1999). 